Cinémas

Revue d'études cinématographiques

Journal of Film Studies

\title{
Intériorité/plasticité. La théorie de la mise en scène de S. M. Eisenstein
}

\section{Maria Tortajada}

Volume 11, numéro 2-3, printemps 2001

Eisenstein dans le texte

URI : https://id.erudit.org/iderudit/024854ar

DOI : https://doi.org/10.7202/024854ar

Aller au sommaire du numéro

Éditeur(s)

Cinémas

ISSN

1181-6945 (imprimé)

1705-6500 (numérique)

Découvrir la revue

Citer cet article

Tortajada, M. (2001). Intériorité/plasticité. La théorie de la mise en scène de S. M. Eisenstein. Cinémas, 11(2-3), 225-252. https://doi.org/10.7202/024854ar

\section{Résumé de l'article}

Se référant à Freud, Eisenstein réfléchit, dans les années trente, à l'idée d' " acte manqué » dans le jeu de l'acteur et l'associe à ce que Stanislavski appelle le « sous-texte ». Dans « Mise en jeu et mise en geste », il s'y réfère pour construire un principe de jeu à travers l'étude détaillée d'une scène de L'Idiot de Dostoïevski. Cette question concerne le passage de l'intériorité de l'acteur-personnage à sa mise en geste dans l'espace. Ni soumis à la rationalité de l'intention de Meyerhold en 1936 (voir par exemple " Chaplin et le chaplinisme "), ni rivé à la quête des " souvenirs affectifs » de Stanislavski, Eisenstein conçoit sa propre théorie de la mise en scène en accordant à l'intériorité, à ce qu'il appelle « interne », un sort très particulier. 


\section{Intériorité/plasticité. \\ La théorie de la mise en scène de S. M. Eisenstein}

\section{Maria Tortajada}

\section{RÉSUMÉ}

Se référant à Freud, Eisenstein réfléchit, dans les années trente, à l'idée d' "acte manqué" dans le jeu de l'acteur et l'associe à ce que Stanislavski appelle le "sous-texte". Dans «Mise en jeu et mise en geste», il s'y réfere pour construire un principe de jeu à travers l'étude détaillée d'une scène de L'Idiot de Dostö̈evski. Cette question concerne le passage de l'intériorité de l'acteurpersonnage à sa mise en geste dans l'espace. $\mathrm{Ni}$ soumis à la rationalité de l'intention de Meyerhold en 1936 (voir par exemple "Chaplin et le chaplinisme"), ni rivé à la quête des "souvenirs affectifs" de Stanislavski, Eisenstein conçoit sa propre théorie de la mise en scène en accordant à l'intériorité, à ce qu'il appelle "interne", un sort très particulier.

\section{ABSTRACT}

In the 1930s Eisenstein took up Freud's idea of the "parapraxis" and considered it in relation to acting and to what Stanislavsky called the "sub-text." In "Acting and Gesturing", Eisenstein set out to construct a principle of acting through the detailed study of a scene from Dostoevsky's The Idiot. Eisenstein's interest lies in the movement from the interiority of the actor-character to his or her gestures in space. Here Eisenstein is neither constrained by the rationality of purpose found in Meyerhold's 1936 article "Chaplin and Chaplinism," nor fixared on Stanislavsky's search for "emotional memories." Instead, Eisenstein conceives of his own 
theory of "mise-en-scène" by confering to interiority, or what he calls "internal," a very special function.

Après Meyerhold, et la biomécanique qui approche le jeu de l'acteur à partir d'un travail sur le corps et la mécanisation du mouvement, après une réflexion et une pratique qui préconisent la "théâtralité», Eisenstein en vient à s'intéresser à un élément qui semble étranger à ses principes premiers. Dans les années trente, on constate un penchant pour ce qui relève de l'intériorité de l'individu, pour ce qui concerne les éléments refoulés et donc l'inconscient. À cette époque, le cinéaste enseigne à l'École de cinéma de Moscou (G'TK, GIK, VGIK) et il conçoit le projet d'un ouvrage intitulé L'Art de la mise en scène (Régissoura) devant reprendre l'ensemble de son travail et de sa réflexion pédagogiques des années 1932-1935. Dans un texte comme "Le retour du soldat du front " $(1970,1989 \mathrm{a}, 1996)$, il fait intervenir le déni, le traumatisme; dans "Mise en jeu et mise en geste" (1986a), il renvoie à Freud et associe la notion "d'acte manqué" à la notion de "sous-texte" de Stanislavski. Cet intérêt pour les valeurs du Théâtre d'art pourrait peut-être s'expliquer par les contraintes du contexte politico-culturel, le réalisme compris à la manière de Stanislavski devenant la référence obligée dans le courant des années trente. Il acquiert cependant une importance esthétique qui échappe aux motivations liées aux rapports de force du contexte artistique et politique.

Au début des années trente, Eisenstein repense la place du travail scénique dans son système de création filmique. Auparavant, il avait abordé la construction du jeu de l'acteur à partir de son expérience proprement théâtrale: dans ses écrits sur le théâtre et dans un article qu'il publie en collaboration avec Sergueï Trétiakov, "Le mouvement expressif» (1998). Il avait touché aussi à la question de l'acteur via le typage, qu'il continue de théoriser en référence au jeu $(1970$, p. $40 ; 1989$ a, p. 405-407; 1996 , p. 89-90). Or, dans son enseignement à Moscou, il consacre une grande part du programme à la mise en scène comme étape spécifique de la création du film (1996, p. 84-90). Cette approche essentielle est clairement distinguée des questions de mise en cadre et de montage. Il s'agit proprement d'instaurer 
une pratique créative concernant le passage d'une histoire, d'une situation, à sa spatialisation et sa mise en action par l'acteur. C'est cet aspect que nous retiendrons ici à l'exclusion des procédés proprement filmiques. Car, sur ce plan déjà, Eisenstein parvient à une théorisation d'envergure.

Les textes d'Eisenstein sur sa pratique à l'école de Moscou se présentent comrne des cours témoignant d'un dialogue avec les étudiants ou comme des essais fondés sur une rhétorique dialogique. Plus que des écrits à visée proprement théorique, ce sont des mises en place progressives de procédés, de concepts et de méthodes de travail, livrés au fil de la discussion avec les élèves ou d'un discours entièrement assumé par le cinéaste. Cette pratique didactique est elle-même une expérience théorique'. À propos du travail avec l'acteur, l'un des textes les plus frappants est sans conteste "Mise en jeu et mise en geste: deux micro-études tirées de LIdiot de Dostoïevski et du scénario de K. Vinogradskaïa ». Tout en suivant trois exemples pour présenter les étapes de travail successives, il en vient à décrire l'entièreté de son système de mise en scène, avec ses enjeux esthétiques, ainsi que les concepts et les principes à l'œuvre dans sa méthode. À travers ces trois exemples - le premier, le scénario de Makogon, et les deux autres tirés de LIdiot - Eisenstein ne nous livre pas seulement une méthodologie pratique, mais une sorte de manifeste théorique déguisé avec, au centre, une définition de la mise en jeu et de la mise en geste, dont la distinction dépasse la question d'une simple sérialisation méthodologique du travail. Pour en saisir toute l'importance, il faut préciser les diverses nuances qui se cachent derrière la notion "d'intériorité", et plus précisément derrière le terme "interne», fréquemment employé. C'est l'intégration de ces notions dans le système eisensteinien qui se joue ici, leur devenir et leur fonction.

Nous allons donc nous livrer à une explicitation du système théorique de la mise en scène tel que synthétisé dans ces exemples. Non seulement ce texte est un transformateur de concepts - il modifie progressivement l'emploi de l'adjectif « interne» —, mais il décrit aussi l'expérience créatrice de la mise en scène comme un processus d'intégration de l'émotion du personnage dans un système qui la réélabore avant de produire un effet sur le spectateur. 
La notion "d'intériorité », couplée avec l'adjectif «interne», est plus que fréquente dans le texte: elle renvoie aux sentiments, aux affects, aux pulsions d'un sujet, et plus particulièrement dans le premier paragraphe, à celle d'inconscient. C'est ainsi que commence le texte:

Freud a, en son temps, fait grand bruit en déclarant (en fondant et démontrant) que le lapsus et la Fehlhandlung [acte manqué] ne sont en réalité ni l'un ni l'autre, mais d'authentiques intentions, forçant une Deckhandlung [acte recouvrant] par laquelle ils sont recouverts, cachés, « refoulés » en raison des conditions, des exigences et des circonstances extérieures (Eisenstein, 1986a, p. 179).

Eisenstein continue en citant Stanislavski, un peu plus loin:

Konstantine Serguïevitch ne proposa pas non plus de théorie de l'«irruption" en surface de ces motifs refoulés. Mais la priorité revient précisément au texte sousjacent qui transparaît à travers le vêtement [l'enveloppe] du texte visible (ibid.).

Cet intérêt pour l'intériorité de l'individu, qui se trouve rapprochée de la notion de "texte sous-jacent", va de pair avec le "refoulé", terme proprement freudien, mais aussi avec le "caché» et le "recouvert " qui renvoient aux émotions dans un registre psychologique distinct de la théorie psychanalytique. Le pendant de la valorisation de cet aspect du sujet est la recherche du "dévoilement », de la «mise à nu» - termes que l'on retrouvera souvent. Se conjuguent donc ici une logique des profondeurs et un principe de surface ${ }^{2}$. La réflexion semble plus proche des fondements du Théâtre d'art que des textes eisensteiniens des années vingt. Eisenstein réfléchissait alors à l'expressivité comme à un conflit de forces physiques sur le corps de l'acteur-personnage. Dans son article de 1923, rédigé avec Sergueï Trétiakov, il rejetait explicitement le "revivre" de la méthode de Stanislavski et concevait le mouvement de l'acteur comme la combinaison de deux actions, l'action réflexe et l'action volontaire (1993a, p. 210-211). Au contraire, chez Stanislavski, l'inconscient est central ${ }^{3}$, car il est présupposé par la 
"mémoire affective" à laquelle l'acteur doit faire appel pour entrer dans son personnage. L'intériorité est alors simultanément l'émotion du personnage et de l'acteur qui, en somme, s'identifient sur scène: la spécification du processus d'adéquation de l'un à l'autre jette les bases de ce qu'on peut appeler une théorie de l'acteur ${ }^{4}$. Malgré les deux références placées en introduction de ce texte sur la mise en scène, Eisenstein se différencie de Stanislavski dans l'utilisation de la notion d'intériorité.

L'intérêt d'Eisenstein pour l'acte manqué proprement dit tient sans doute au privilège accordé à l'action dans ce phénomène de comportement ${ }^{5}$ car c'est, nous le verrons, sous cet angle qu'il met en place un processus faisant intervenir l'inconscient. On le constate dès le début du texte, lorsqu'il explique la fonction de l'intériorité via le "réflexe", qu'il associe à la pratique de l'hypnose ${ }^{6}$. Ce rapprochement en dit long sur la parenté de la méthode d'Eisenstein exposée ici avec ses positions des années vingt: le réflexe était alors saisi comme une pure action et jouait un rôle essentiel sans pour autant renvoyer à un processus psychique. Sa reprise, dans les années trente, pour parler de l'inconscient, renvoie ce dernier à une problématique de l'action.

Par ailleurs, lorsque Eisenstein prend en compte l'intériorité, il considère, sans aucune ambiguïté, l'inconscient du personnage. Il ne s'intéresse pas à ce que nombre de théoriciens du théâtre, à commencer par Diderot, présentent comme la base d'une définition du jeu: le lien de dédoublement - comme chez Meyerhold - ou d'identification - comme chez Stanislavski - entre acteur et personnage. Dans sa réflexion sur la mise en scène, on ne trouve pas à proprement parler de théorie de l'acteur ${ }^{7}$. S'il reprend les termes d'incarnation et d'expression qu'utilise Stanislavski, c'est pour leur donner un sens qu'il réélabore en accord avec sa propre théorie. Nous devons essayer de comprendre ces glissements et déplacements. Néanmoins, il nous faut partir de cet élément apparemment paradoxal dans le système eisensteinien : la place prééminente accordée à l'intériorité.

\section{L'action et le motif dans la mise en jeu}

Lorsque Eisenstein aborde un exemple pour faire comprendre ce qu'il entend par mise en jeu, l'intériorité désigne, dans son 
discours, les émotions et les intentions cachées d'un personnage. Ce sera le cas du premier exemple analysé, tiré du scénario de Makogon:

Un jeune homme a signé l'acte de mariage avec une jeune fille et l'amène pour la première fois dans sa chambre. Fermant la porte derrière lui, il lui explique que la porte est nantie d'une serrure française (1986a, p. 182).

Eisenstein s'intéresse à ce qui pousse le jeune homme, d'une part à fermer la porte à clef et, d'autre part à fournir cette explication technique sur la nature de la serrure. Quelles intentions sont à l'origine de ces deux actes? Ce qui est visé, c'est la motivation intérieure de l'action. L'acte manqué, auquel le début de l'article est consacré, retient en effet l'attention d'Eisenstein parce qu'il met en place un décalage, une inadéquation entre le motif visible d'un acte et ce qui inconsciemment le provoque. Le caché, en somme, vaut essentiellement pour l'impulsion qu'il fournit, pour la causalité qu'il met en place. C'est une manière de faire entrer l'espace intérieur du personnage dans une logique de l'enchaînement des actions.

Dans cet exemple, Eisenstein formule le motif qu'il recherche en partant du scénario: compte tenu de la psychologie du personnage masculin, il apparaît - et Eisenstein passe rapidement là-dessus dans ce cas-ci - que le jeune marié considère la femme comme sa propriété : par conséquent, il l'enferme. C'est là le "motif direct" (p. 182) de l'action, motif peu avouable qui se voit dissimulé sous une explication technique: il est en quelque sorte "neutralisé". En dévoilant le motif caché de l'action, Eisenstein pose les rapports logiques mais ne définit pas encore le jeu.

Le principe du cinéaste est de déployer le lien entre le motif et l'action comme un processus. Il serait erroné de croire qu'il s'agit là d'une simple causalité. Eisenstein récuse l'organisation de l'action selon un rapport d'intention et d'exécution, que l'intention soit cachée ou non d'ailleurs:

À la différence de la pratique quotidienne où tout se résume à la réduction et l'élimination des maillons, le 
secret d'une mise en scène efficace consiste à déployer sur scène un processus entier. (Voir mon analyse du mouvement de recul qui, en son nux [noyau], dévoile toute une méthode - des deux termes de la logique, le processus est renvoyé aux trois termes déployés de la dialectique; non pas: intention-exécution, mais: négation de la négation) (p. $182^{8}$ ).

En impliquant un troisième terme - le motif caché derrière un autre qui n'est qu'apparent - la notion d'acte manqué permet d'introduire un processus dialectique. Il s'agit pour Eisenstein de produire un effet sur le spectateur. Pas question de chercher cela du côté du travail de l'acteur sur lui-même, dans une forme d'expressivité qui se définirait par une causalité simple: faire jaillir l'émotion cachée et la laisser s'inscrire sur le visage de l'acteur ou dans ses gestes comme le veut la méthode de Stanislavski. Celle-ci est fondée sur le passage direct de cet élément essentiel qu'est l'émotion: passage de l'intériorité de l'acteurpersonnage à la surface visible du corps d'où elle pourra "atteindre" directement le spectateur". L'essentiel du travail, dans cette théorie, c'est de retrouver l'émotion, de la revivre. Une fois qu'elle "est là ", cependant, elle se donne à voir "naturellement " telle quelle. Pour Eisenstein, l'accès direct à la visibilité et son effet sur le spectateur ne vont pas de soi. C'est dans le processus que se révèle cette intériorité, considérée dans son aspect purement dynamique. Or, la mise en évidence du caché nécessite la participation de l'actrice:

Nous avons dit ensuite que les mots concernant les propriétés de la serrure sont un camouflage distrayant du motif réel du jeu autour de la serrure. Pour qu'apparaisse ce camouflage, il faut que surgisse sa nécessité. La nécessité de dissimuler le motif véritable. De cacher le motif réel sous une "information " apparemment innocente sur les propriétés de la serrure. Où chercher le prétexte d'une telle nécessité? Bien sûr, dans le jeu de la femme (p. 183; c'est nous qui soulignons).

Le moment est essentiel. Pour laisser paraître l'intériorité, c'est-à-dire la motivation profonde du jeune homme, il faut que la femme joue. Dans la perspective de Stanislavski, cela semblerait 
pour le moins paradoxal! Le lieu où s'expriment les énergies inconscientes du personnage, c'est d'abord le corps de l'acteur qui les "vit». Avec Eisenstein, on échappe à l'individu: l'expressivité ne se pense que dans le collectif ${ }^{10}$. Il ne s'agit pas de faire remonter des profondeurs de l'inconscient ce qui y est enfoui, mais de motiver son apparition par l'intervention d'un autre personnage, par une cause extérieure. Ce qui prime c'est la logique de surface, le jeu entre les personnages. En somme, Eisenstein procède à la mise à plat de ce qui aurait pu se cantonner dans une psychologie des profondeurs, et fonde ainsi le processus dialectique de l'action et de la motivation, comme le veut l'exemple.

Entendant tourner la clef dans la serrure, la femme porte son attention à ce geste sans le moindre soupçon. La motivation du jeune homme reste bien sûr cachée. Pourtant l'intérêt de la jeune épouse rend nécessaire l'explication technique sur la serrure: "Ce manque de soupçon servira d'autant plus élégamment de raison à la réplique camouflante du jeune homme qui tâche de masquer moins le soupçon réel de la femme que sa supposition qu'elle doit en nourrir un" (p. 182, c'est nous qui soulignons). Eisenstein définit donc une autre cause aux explications fournies par le jeune marié. Pour mettre en place le jeu, il ne se contente pas du motif caché, mais fait intervenir dans le processus un second motif, qui échappe à la logique inconsciente du seul individu. L'introduction de ce motif vient soutenir la dynamique de l'ensemble, et participe au processus dialectique de l'enchaînement des actions. La négation de la négation : ainsi, le motif direct de l'acte du jeune homme — la volonté d'enfermer la propriété acquise - est nié dans son discours sur les aspects techniques de la serrure. Mais ces mêmes mots sont amenés par une autre cause, extérieure au personnage masculin: l'attitude de la jeune femme qui annule cependant par son innocence la nécessité du déni impliqué dans les explications de son mari. En somme, le commentaire technique est la résultante de deux motifs qui s'articulent, et non plus d'un seul, purement intérieur et émotionnel. On comprend alors que l'expressivité ne se pense pas uniquement à travers le travail de l'acteur lui-même, mais dans le rapport que le personnage entretient avec les autres. Le 
jeu, c'est justement ce dispositif complexe de motivations concurrentes qui constituent l'action.

Le processus dialectique n'est pas sans effets sur le système des motivations et le statut de l'intériorité. Le jeu vise constamment à tirer la dynamique des actions vers un enchaînement de surface, en faisant passer les motifs vers l'extériorité, en les faisant assumer par les autres acteurs. En somme en minimisant la psychologie des profondeurs au profit d'une logique du «tout visible». Eisenstein conclut cependant en soulignant l'importance de l'intériorité du personnage:

L'exemple cité nous a dévoilé la manière dont un personnage et son conflit intérieur typique deviennent visibles et tangibles par lincarnation de ces deux aspects en éléments d'une situation effective: en un nouveau maillon de "scénario" qui se développe à partir de la nécessité d'incarner de façon tangible le jeu secret des motifs dans l'interprète (p. 185 ; c'est nous qui soulignons ${ }^{11}$ ).

L'intériorité psychologique du personnage est ici clairement désignée comme élément central du jeu. Cependant, l'utilisation du terme d' "incarnation" ne saurait renvoyer à la prise de possession physique et morale de son personnage par l'acteur. L'exemple l'a montré, l'incarnation ne peut se faire que par le jeu, car ce qui est incarné, c'est le conflit des motifs: l'incarnation est la dialectique des actions rendue par le jeu. Cela est bien différent du conflit intérieur qui s'exprime en dramaturgie classique par le dilemme: une tension consciente chez le personnage entre deux choix, deux motifs, deux actions. La différence ne relève pas seulement de ce que l'exemple d'Eisenstein travaille avec l'inconscient. Elle tient beaucoup plus encore au fait que la tension entre les motifs se déplie dans le processus de la mise en jeu; elle échappe par-là au seul sujet.

Dans la citation que nous venons de mentionner, il reste néanmoins une ambiguïté : le conflit est bien à l'intérieur du personnage. Ce type d'ambiguïté est le propre de ce texte et l'indice de la transformation que subissent les notions d' "interne" et de "caché ${ }^{12}$ ". Cela apparaît de manière manifeste quelques paragraphes plus loin. Eisenstein parle plus précisément des 
mouvements des acteurs, ce qui annonce déjà la partie sur la mise en geste:

[...] tous ces facteurs se révéleront aussi humblement associés à la tâche de donner une incarnation tangible au motif et à l'intention; à cette seule différence qu'ici, les éléments de répartition spatiale des différents membres d'une personne entre eux, de la personne dans son ensemble avec un partenaire et, enfin, de la personne en interrelation avec l'espace, offriront un tracé assez lisible pour dévoiler le sens interne et le jeu des motifs au sein des personnages (p. 185 ; c'est nous qui soulignons).

Cependant, si les «motifs au sein des personnages" ont été clairement explicités jusqu'ici, le "sens interne" reste à définir.

\section{Le «sens interne» et le principe de surface}

L'exemple de la tentative d'assassinat du prince tirée de L'Idiot en fournit l'occasion. Il s'agit ici de faire la démonstration de la mise en geste. La difficulté est qu'Eisenstein ne cesse de renvoyer à la mise en jeu, ce qui rend la distinction difficile, même si en définitive il est tout à fait possible de la saisir clairement. L'explication nécessite cependant une redéfinition de la notion d'action, puis de "l'action négative", qu'il faut distinguer de "l'action inversée". La deuxième difficulté de cet exemple relève de la nature des actions qu'il met en place. Son originalité tient, comme nous le verrons, au fait que, au cœur de la situation, il y a ce qu'on peut appeler une non-action: le prince agressé ne se défend pas. L'exemple est entièrement construit sur une sorte "d'euphémisation» des actes, qui rend forcément complexes les articulations entre les diverses actions et difficile la tentative de cerner leur nature. À cela s'ajoute une procédure sensiblement différente dans le traitement de l'exemple. Contrairement au cas précédent où le motif caché était visé directement et défini d'emblée, ici, tout en l'impliquant, Eisenstein donne la priorité à la mise en évidence des actions elles-mêmes dans l'organisation de la réflexion. La tendance se poursuit donc d'accorder le privilège à l'explicite, au visible, par rapport à ce qui est intérieur.

Eisenstein choisit l'épisode où, après avoir longuement suivi le prince Mychkine, Rogogine tente de l'assassiner: dissimulé 
dans une niche, il surgit soudain devant lui. Citons un extrait du texte de Dostoïevski retenu par Eisenstein (p. 186) :

\begin{abstract}
Alors, les deux yeux de l'après-midi, les mêmes yeux, croisèrent soudainement les siens. L'homme caché dans la niche avait fait un pas pour en sortir. Pendant une seconde tous deux restèrent face à face, se touchant presque. Brusquement le prince empoigna l'homme par les deux épaules et l'entraina dans l'escalier, vers le jour, pour mieux le dévisager. Les yeux de Rogogine étincelèrent et un sourire de rage crispa ses lèvres. Il leva sa main droite dans laquelle brillait un objet. Le prince n'eut pas l'idée de le retenir. Il se rappela seulement plus tard avoir poussé ce cri : - Parfene, je ne puis y croire!
\end{abstract}

Il sera alors victime d'une attaque d'épilepsie.

Dans la chaîne d'actions qu'il établit (p. 189), Eisenstein isole justement le très bref moment où le prince ne réagit pas, ne se protège pas, malgré l'agression frontale qu'il subit: il "n'eut pas l'idée de le retenir». L'incohérence de la réaction est définie comme un "cornportement "inverse des normes reçues de conduite" "(p. $189^{13}$ ). Pour en expliquer le principe, Eisenstein se réfere à $\mathrm{I}$. Tourguéniev qui reproche à Dostö̈evski d'utiliser un "lieu commun inversé" en toute situation: une personne qui a peur blêmit et s'enfuit; chez Dostoïevski, elle rougit et reste sur place. Reprenant ce procédé, Eisenstein le fonde sur le caractère du personnage, sur sa psychologie, qu'il formule à partir de son interprétation dù roman. Le prince se définit lui-même comme quelqu'un qui ne dispose pas du geste qui convient: "Je n'ai pas le geste... j'ai toujours le geste contraire..."; son inadéquation au monde apparaît à diverses occasions. Ce qui intéresse Eisenstein, ce sont les motifs intérieurs qui justifient l'inversion des actes du prince.

Pourtant, Eisenstein ne se lance pas immédiatement à la recherche du motif caché. Son texte continue par la description de l'action "maillon après maillon" dans lequel il en isole le cinquième, que nous venons de mentionner. Ce moment pose justement un problème: comment indiquer en effet ce que le prince "ne fait pas"? 
Cela sonne merveilleusement dans une narration. Cela donne une nuance raffinée à la description; mais il lui manque malheureusement une transposition directe en action, en geste ou en représentation (p. 190).

Eisenstein se lance alors dans une enquête. Ce n'est pas le motif qu'il vise, mais l'action de substitution de l'action inversée: «[...] ce que faisait le prince au moment de ne pas arrêter la main de Rogogine" (p. 192). Avant d'aborder ce problème, Eisenstein fait un détour par la définition de "l'acte négatif». On pourrait croire qu'il s'agit là d'un élément nécessaire à la compréhension du rapport entre acte inversé et acte de substitution, mais le lien n'est pas direct. Ce n'est que plus tard que cette définition sera utile. Nous y reviendrons.

Pour le moment, Eisenstein cherche l'acte de substitution à travers une lecture du texte de Dostoïevski qui établit le caractère et la psychologie du personnage. Il s'arrête à ces yeux qui poursuivent le prince sans qu'il ne les reconnaisse vraiment:

\footnotetext{
Rigoureusement parlant, l'image de Rogogine porteur et possesseur des mêmes yeux que ceux qui le poursuivent de page en page dans la description qui précède est loin encore d'avoir pénétré dans la conscience du prince Mychkine. Et plus exactement, les yeux de Rogogine sont déjà "entrés" dans le prince mais ne sont pas encore parvenus à la conscience et n'ont pas abouti au dévoilement de leur propriétaire (p. 191).
}

Au moment de l'agression, l'identité du suiveur ne lui est pas encore apparue, mais c'est là qu' elle va se révéler :

[...] ce moment [où le prince se persuade d'avoir affaire à Rogogine] se dissimule aussi sous cette brève ligne où [il] ne pense pas à arrêter la main au couteau qui se lève, mais, c'est chose claire, pense, se convainc absolument que cet homme devant lui doit être Rogogine (p. 192).

A travers l'analyse psychologique, l'action de substitution s'impose: il s'agit d'un acte de reconnaissance. Un acte dont la force n'est pas des plus manifeste, puisqu'il s'agit d'une perception et d'une intellection, non pas d'un geste capable de trans- 
former un état concret du réel. C'est en ce sens que nous pouvons parler pour cet exemple d'une "euphémisation" de l'action; cela a l'avantage de démontrer qu'il est possible de trouver une action dans toute situation, et que, par conséquent, il est légitime de décrire le monde, et donc la mise en scène, selon ce critère. La position théorique et méthodologique d'Eisenstein s'en trouve renforcée.

En cherchant l'acte de substitution, Eisenstein impose deux actes à la fois, et change la nature de sa série d'exemples: dans l'acte manqué ou dans l'exemple tiré de Makogon, deux motifs sous-tendent en effet un seul acte. Pourtant, comme dans ces deux cas, la situation de l'agression est bien structurée par un décalage entre motif et action. À travers l'analyse du roman, Eisenstein fait ressortir le motif psychologique et intérieur de l'acte de substitution, qui devient — nous devons le déduire de la démonstration - le motif caché du comportement inattendu: si Mychkine est obsédé par l'acte de reconnaissance, c'est qu'il refuse malgré lui d'admettre qu'il s'agit de Rogogine. "Il veut si peu y croire que déjà convaincu, il crie: "Je ne puis y croire" "(p. 191): il se voit ainsi contraint à l'acte d'identification, et détourné d'un réflexe défensif. Un acte manqué peut apparaître comme une véritable perte de maîtrise de l'individu sur ce qu'il fait. C'est ce qui retient un cinéaste comme Lev Kouléchov dans son film La Journaliste ${ }^{14}$, où les personnages jouent la maladresse. Dans cet exemple d'Eisenstein, la situation est sensiblement différente: grâce à l'action de substitution est mise en scène une sorte de prise de conscience du personnage, exclue du processus de l'acte manqué. Si c'est bien "malgré lui" que le prince ne se défend pas, il n'en est pas moins impliqué simultanément dans un acte d'identification, ce qui en soi est déjà un début de maîtrise; la différence de conception est notable, même si le moment est présenté comme un "choc", comme une « reconnaissance abasourdie" (p. 193).

En faisant intervenir deux actions, Eisenstein recrée un processus dialectique: une tension entre deux motifs, l'un extérieur - la nécessité de se défendre —, l'autre intérieur au personnage, le refus de reconnaître, qui impose la concentration sur le regard à la place du geste défensif. Jusqu'ici, nous avons parcouru un 
chemin analogue à celui qui était exposé dans le premier exemple: en articulant actions et motifs dans une dynamique dialectique, le texte nous livre la mise en jeu d'une situation. Remarquons qu'Eisenstein utilise un scénario ou un roman traité comme tel pour établir le motif caché. En somme, s'il y a un dévoilement de l'intériorité du personnage, il se fait à travers une herméneutique du texte littéraire, et non pas grâce à un travail sur l'inconscient de l'acteur. Pour ce dernier, le caché est justement ce qui est donné, ce qui est connu à partir du travail textuel que fournit le metteur en scène. Ce paradoxe apparent souligne la possibilité d'un dévoilement, et fait apparaître l'intériorité et l'inconscient comme des entités toujours accessibles.

La nouveauté de l'étape théorique est introduite dans la suite de la réflexion proposée par Eisenstein. Pour exprimer l'action de substitution - la tentative de reconnaissance -, il isole le voir et le toucher. Il décrira donc l'attitude du regard du prince et le mouvement de sa main se posant malgré lui sur celle de Rogogine, et même sur le couteau, comme pour le reconnaître et non pour se défendre.

Certes, la main qui, dans son errance (toute l'attention se portant sur l'œil!), "ne sait ce qu'elle fait» se posera à côté de la lame, effleurant ou presque le tranchant scintillant et soigneusement effilé. Jetons un coup d'œil sur le raccourci ainsi créé. Le prince s'est avancé tout contre Rogogine. Sa main est posée sur la main qui lève le couteau en arrière pour frapper. Et quel serait le tracé du mouvement si le prince "songeait " à l'arrêter [la main S.M.E.]? Un bond vers Rogogine (en s'avançant tout contre lui). Et... la main du prince sur la main (ou le couteau) de Rogogine. En d'autres termes: un contour spatial identique. Quand au contenu interne du mouvement - deux actions diamétralement opposées - de toute l'étendue des $180^{\circ}$. [...] Les « fenêtres éclairées" et les "fenêtres sans lumière" (p. 194).

Le geste est identique dans les deux cas même s'il implique deux actions différentes: se défendre et reconnaître. C'est en quelque sorte la mise en place d'un "acte négatif ". Le moment théorique qui concernait cet acte passait par l'exemple des fenêtres. Il définissait l'action négative comme celle qui «en conser- 
vant l'apparence d'un contour identique à la première est diamétralement opposée dans l'essence et dans son contenu» (p. 191). Ainsi, si on montre "une suite de quelques fenêtres éclairées et soudain une fenêtre sombre: il y a bien des chances pour que l'on lise précisément cette fenêtre comme "une fenêtre sans lumière" " (p. 190)). La forme est la même dans les deux cas, mais ce qui est désigné est le contraire exact. La dialectique est encore la raison du processus: un effet est produit grâce à la tension entre deux éléments contraires.

Pour l'exemple de Mychkine, l'opposition réside dans «le contenu interne du mouvement». Faut-il comprendre "contenu interne» comme l'intériorité du personnage? Certainement pas: Eisenstein le précise, ce contenu est composé des "deux actions diamétralement opposées", se défendre/reconnaître. Or, jusqu’à présent, tout ce qui relevait du caché appartenait au motif inconscient du personnage. Et, dans la mise en jeu, il était clair que le motif intérieur était la cause d'un acte qui, lui, n’avait rien de voilé puisqu'il était visible dans le comportement du personnage. Cette fois, au contraire, les actions se retrouvent au niveau interne en regard des gestes eux-mêmes qui les signifient: voir, toucher. Eisenstein est en train d'opérer ici un déplacement radical dans l'usage de l'adjectif "interne", essentiel à la distinction de la mise en jeu et de la mise en geste.

Bien qu'ambigu, ce déplacement ne relève pourtant pas d'un manque de cohérence. On comprend mieux ce qui se produit ici au niveau théorique si l'on constate qu'Eisenstein définit l'action selon deux niveaux. Il y a une action-comportement - reconnaître, se défendre - dont le rapport aux motivations intérieures du personnage définit la mise en jeu. Il y a par ailleurs une action-geste qui donne forme à l'action-comportement, qui la matérialise dans la mise en geste. Cette dernière trouve son "contenu interre" dans l'action-comportement. Plus précisément : le contenu est donné par le jeu lui-même - ce rapport complexe élaboré précédemment entre les motifs et les actionscomportement. Lorsque Eisenstein parlait d'actes inversés, il désignait le premier stade, lorsqu'il utilise le terme d'action négative, c'est pour préciser le rapport entre les actions-geste. Les deux niveaux ne se recouvrent pas, même si les deux sont 
structurés par des rapports dialectiques: de même que la tension entre deux motifs produit un acte-comportement, la tension entre deux de ces actes sous-tend l'action-geste (voir figure 1).

Figure 1

Schéma (partiel) du deuxième exemple:

l'agression du prince dans L'Idiot.

Mise en jeu et mise en geste: les deux niveaux dialectiques

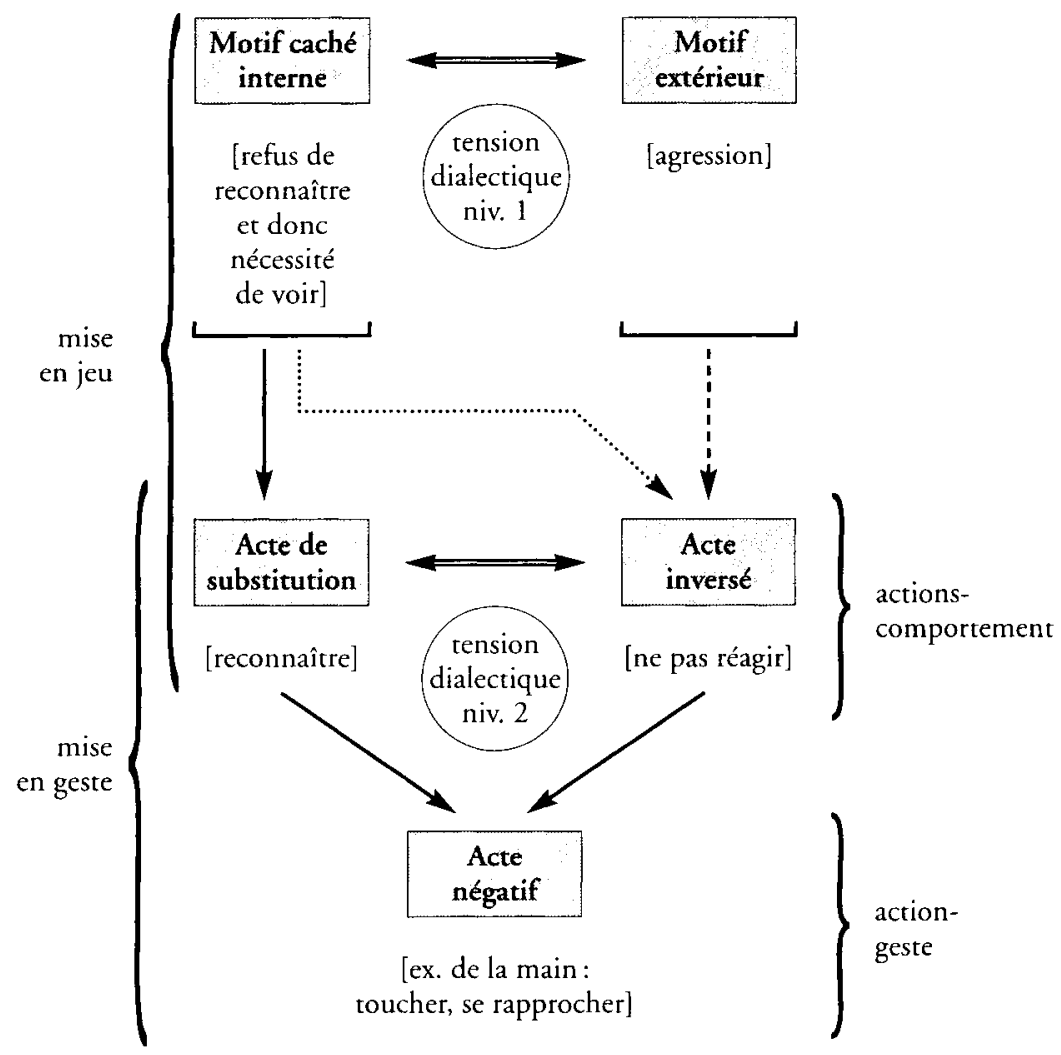

$\longrightarrow$ Lien de causalité explicite Lien de causalité implicite Lien de causalité inadéquat

Si on confond les deux niveaux, on manque le saut essentiel qui s'opère dans la méthode d'Eisenstein et qui déplace l'emploi de "caché" et d' "interne". Nous parlions d'intériorité, de motif caché dans le sens psychologique; maintenant, dans la mise en 
geste, «interne" renvoie à un "contenu ». Le rapport est d'ordre sémiologique, même si ce terme n'est pas à prendre dans le sens fort: il ne s'agit pas d'une relation sémantique surdéterminée. Mais le changement de registre est significatif dans la méthode que propose Eisenstein. Les deux sens d' « interne» coexistent sans qu'il n'en explicite l'ambiguïté ${ }^{15}$. Ce saut, comme nous le verrons, met en place le début d'une transformation conceptuelle, mais aussi fonde les principes de la mise en scène. Il est au cœur du processus qui intéresse le cinéaste dans le travail avec l'acteur.

Cette transformation s'accompagne d'une relativisation de l'intériorité psychologique dans le système de la dialectique, qui confronte l'inconscient à d'autres motivations et à plusieurs actes concurrents. En effet, le motif intérieur n'est pas seul à régir la dynamique. Cette relativisation de l'intériorité psychologique apparaît aussi dans la tendance - repérée déjà dans le premier exemple - à rabattre le motif intérieur sur un fonctionnement de surface. Cela est frappant dans les actions-geste qu'Eisenstein présente ensuite.

Il va décrire ainsi le voir et le toucher en montrant leur enchaînement selon une nécessité physique: l'ensemble est traité du point de vue de la plasticité. C'est d'ailleurs à ce stade qu'Eisenstein illustre son propos par des dessins. Tout est régi par les lois de l'enchaînement qui s'établit selon une logique générative spatiale. Si le motif caché et psychologique est essentiel dans le processus, la logique des actions-geste en prend le relais suivant une dynamique de surface. Ainsi, Eisenstein orchestre le moment de l'agression jusqu'à la crise du prince en soulignant la production d'un mouvement par celui qui le précède. Le prince voyant enfin Rogogine:

Et voici l'horreur parvenue à une apogée; en cercles concentriques, elle se brise dans un zigzag de la main: "Je ne puis y croire!" [...] Il tente vainement de rompre le fil de fer du parcours de pupille à pupille - par le truchement rapide du bras droit dessinant les zigzags de la négation, les jambes emportent le corps qui se pétrifie des yeux aux pieds. Dans son essor le zigzag se transforme en nervosité. Sa trajectoire est comme le début hésitant d'un "non" d'horreur non encore littéral [...] Et voici déjà que sarrache ce "je ne puis y croire

Intériorité/plasticité. La théorie de la mise en scène de S. M. Eisenstein 
[souligné par S.M.E.] !» même (p. 198-199; c'est nous qui soulignons).

Cette tendance à la causalité de surface dans l'enchaînement des mouvements entre eux se confirme lorsque Eisenstein en vient à motiver le soubresaut du prince. Eisenstein définit ici une rupture dans la série de mouvements, décrits jusque-là selon la logique formelle des cercles concentriques, car ce qui suit est un mouvement de recul devant l'horreur. Le changement est radical dans l'orientation du geste. C'est alors qu'il explique :

\begin{abstract}
Une rupture aussi brusque du système entier des manifestations expressives ne peut avoir lieu sans un nouveau choc impulsif. Sans impulsion nouvelle, le mouvement d'un système, d'une inertie en progrès si intense, ne peut se muer soudain en nouveaux aspects de comportement. Et, compte tenu de la stupeur de la personne horrifiée elle-même, cette impulsion ne peut alors être intérieure, des conflits internes ne peuvent dicter de rupture dans le domaine des manifestations extérieures, signalant la victoire de certains motifs (en lutte) sur d'autres (p. 200 ; c'est nous qui soulignons).
\end{abstract}

C'est le contact du couteau, "du métal froid de la lame", qui déclenche le choc instantané et inverse le mouvement dans l'espace. Dans la dynamique de l'action et du geste, l'impulsion extérieure prend le dessus et se substitue comme principe moteur au motif interne. La visibilité, l'enchaînement à la surface, en somme, prime sur une psychologie des profondeurs. Nous passons rapidement sur les détails de la description des différents moments, de ce qu'Eisenstein appelle les maillons de "la chaîne du jeu " (p. 201). Il nous faudrait nous attarder encore à une autre impulsion extérieure et au contre-accent (p. 201), à la mise en place d'une nouvelle action inversée (la main de Rogogine se baissant, p. 202). Mais nous avons dégagé les deux principes essentiels: l'attraction de l'ensemble du système vers une logique de surface; une redéfinition de ce qui est considéré comme interne, avec le maintien ambigu des significations psychologique et sémiologique ${ }^{16}$. 


\section{Représentation et image}

Dans la transformation de ce qu'il désigne comme "interne", Eisenstein ne s'arrête pas là. Le dernier exemple opère un nouveau déplacement en abordant la mise en scène. Également tiré de L'Idiot, il est cependant traité de manière totalement indépendante de celui qui précède. La scène qui intéresse Eisenstein montre le prince et Rogogine, amis et rivaux, se dirigeant vers l'appartement de la femme qu'ils aiment, où elle gît déjà morte. Ils marchent chacun sur un trottoir, parallèlement, séparés par la largeur de la rue et par tous les conflits qui les habitent. Pourtant, ils se retrouveront ensemble auprès de celle qui les a désunis. Eisenstein s'appuie sur une description fournie par A.L. Volynski, qui rend évidentes les "particularités de construction de cette scène" (p. $204^{17}$ ). Ce qui retient le cinéaste, c'est la tension qui lie les deux personnages dans cette situation et la manière dont elle est rendue:

Le mouvement dans une direction unique. Par des voies non encore convergentes. Parallèles. Des voies finalement propres à converger, à se rencontrer, à se réunir au mépris de la logique mathématique mais en vertu de celle de sentiments humains malgré tout après rapprochements et séparations, regards jetés l'un sur l'autre par-dessus l'épaule ou mouvements l'un vers l'autre et vice versa (p. 205).

Dans cet exemple, pas d'allusion à l'acte manqué; pas de réflexion non plus sur les motifs cachés et décalés des actions d'un personnage. Pourtant, la dimension psychologique est centrale. Elle est cependant abordée d'emblée sous l'angle du collectif: sont visées "les relations mutuelles internes» (p. 204) - nous trouvons aussi l'adjectif "profondes" - des deux personnages. II s'agit là effectivement d'un usage élargi de l'intériorité qui impose d'appréhender le caché, voire les inconscients respectifs des deux hommes, comme un ensemble d'interrelations. La formulation d'Eisenstein, ici, maintient un certain flou, et semble faire référence à la fois aux motifs des personnages et à ce qu'il a appelé le jeu, l'interaction dialectique des motivations et des actes de plusieurs individus. En somme à ce que nous avons vu dans les exemples précédents. Retenons cependant qu'Eisenstein place 
le psychologique au centre du processus qu'il décrit pour mieux le soumettre à un dévoilement: comme y parvient Dostoïevski, le metteur en scène doit révéler " (à demi) les relations mutuelles secrètes et profondes existant entre les personnages" (p. 205).

Lambiguité est maintenue quant à l'emploi d'«interne" lorsque Eisenstein explicite le schème de ce troisième exemple, construit selon deux plans: celui du quotidien et celui qu'il appelle «le second plan", qui n'est ni allégorique ni symbolique. Ce dernier renvoie au "texte sous-jacent ", qu'Eisenstein mentionne déjà dans l'introduction en rapport avec l'acte manqué et la référence à Stanislavski. Il commente à propos de ce troisième exemple:

Mais l'acte lui-même et la forme (souvent le dessin) de
son exécution possède, outre le niveau direct et quoti-
dien de son exposition, un second plan - un "texte
sous-jacent" si l'on veut - celui de son vrai sens (de
son vrai contenu); s'il transparaît avec autant de net-
teté, c'est précisément grâce au profil plastique d'une ac-
tion courante possible dans toute une série de formes
également quotidiennes, mais présentée dans le seul
dessin possible pour l'expression d'un contenu de pen-
sée donné (p. $203^{18}$ ).

Le texte sous-jacent, le second plan, c'est le «sens", le "contenu" qui doit "transparaître" grâce à une forme particulière: "le profil plastique" de l'action commune aux deux hommes. Nous sommes bien dans une problématique du dévoilement du caché, de ce qui gît sous les apparences (du sous-jacent). Nous avons déjà vu ce processus: le sens interne est ce qui est défini à travers la dialectique de la mise en jeu. Il ne s'agit plus à proprement parler d'une intériorité psychologique, mais d'un contenu qui relève d'un registre sémiologique.

Eisenstein définit ici deux sortes de contenus à la plasticité: celui du quotidien, qui se donne d'emblée, et un autre, qu'il faut découvrir sous ce dernier - un deuxième plan. Or, le rapport sémiologique que nous venons de poser se voit perturbé. Eisenstein introduit deux notions qui se complètent dans son système de pensée pour les appliquer à la mise en scène: la représentation et l'image (globale), qu'il relie respectivement aux deux plans de contenu: 
J'emploierai les dénominations de "représentation" et d'«image". Je dirai qu'en tant que représentation, le déplacement sur deux trottoirs est une mesure de précaution. Tandis qu'en tant qu'image, c'est une magnifique mise à nu - par la mise en scène - de l'essence des liens mutuels de ces gens évoluant vers le dénouement de leurs relations dans le roman [...] (p. 205).

À suivre cet extrait, la forme plastique (la marche sur les deux trottoirs) - en quelque sorte la mise en geste de la mise en jeu - peut renvoyer à un sens quotidien, qui reste un sens «direct ", immédiat. Il s'agit alors d'un phénomène de représentation. À un deuxième niveau intervient l'image, ce contenu caché qui se dévoile dans la mise en scène. Le second niveau implique le premier, car il n'y a pas de mise en scène sans la conjonction de l'image et de la représentation:

Ces deux plans, chacun dans la rigoureuse logique du domaine qu'il recouvre - le plan de la représentation et le plan de l'image - sont les deux éléments indispensables dans toute mise en scène réellement expressive (p. 206).

Nous atteignons la fin du parcours théorique, la définition de la mise en scène suivant ses différents stades. Mais en introduisant les termes de représentation et d'image, Eisenstein actualise les valeurs qui leur sont associées ailleurs. Elles impliquent une modification dans le rapport sémiologique construit sur le modèle des deux faces du signe, qui se traduisait par les notions de sens et de contenu articulées à la forme et à la plasticité. Si Eisenstein définit en général la représentation comme "la forme, l'apparence plastique (Albera dans Eisenstein, 1986a, p. 278) - dans ce texte, il s'agit de l'apparence plastique en tant qu'elle a un sens direct - , il fait correspondre l'image avec le texte sous-jacent, le contenu, ou le thème de base, en impliquant une valeur active dans la mise en place du sens:

Mais le vrai "contenu» (le texte sous-jacent) de cette mise en scène inhabituelle n'est autre que l'image esquissée des relations mutuelles internes... (p. 204, c'est nous qui soulignons).

L'image est le produit de la forme - elle est "esquissée" produit qui se construit à travers la représentation; mais, nous 
l'avons vu, elle est aussi le contenu caché. Entre contenu caché et sens produit, une différence est manifeste: le premier met en jeu une structure de signification vue sous un angle statique, comme un état où le signifié est donné, déjà là ; le second est au contraire dynamique, il implique une action créative, et introduit une direction dans le processus de construction du sens.

Si on précise encore la définition de l' "image", la distinction s'accentue. Il faut ici mettre en parallèle la mise en scène et le jeu de l'acteur avec la réflexion d'Eisenstein sur le montage. C'est ce qu'il opère lui-même dans "Montage 1938":

Qu'est-ce, en effet, que le montage ainsi conçu? Dans le cas présent, les éléments n'existent plus comme quelque chose d'indépendant, mais comme une représentation particulière d'un unique thème d'ensemble qui les traverse tous également. La juxtaposition de ces détails particuliers dans un certain mode de montage appelle à la vie, rend perceptible l'ensemble qui a engendré chaque partie, elle les relie les uns aux autres dans un tout, dans cette image synthétique où l'auteur et, après lui, le spectateur, revivront le thème en question (Eisenstein, 1958, p. 71).

Eisenstein insiste en même temps sur le fait que, d'une part, l'image est le tout de chaque partie, que la partie contient toujours le thème unique, et que, d'autre part, l'image synthétique - ou globale - est un processus dynamique et créatif. Ce second aspect est impliqué aussi dans le jeu de l'acteur:

Je parle de l'image de l'œuvre entière aussi bien que de l'image d'une scène isolée. Et l'on a absolument le même droit de parler dans le même sens de la création d'une image par l'acteur. L'acteur a exactement la même tâche à réaliser : il doit traduire en deux, trois ou quatre traits de caractère ou de comportement les éléments de base qui, juxtaposés, recréeront le personnage, l'image totale voulue par l'auteur, le metteur en scène et l'artiste lui-même. Qu'y a-t-il de remarquable dans cette méthode? Avant tout son dynamisme, le fait que l'image recherchée n'est pas donnée, mais qu'elle surgit, quélle naît (1958, p. 84). 
Dans le texte "Mise en jeu et mise en geste", cette caractéristique dynamique de l'image globale est présente également. Mais l'image y est décrite simultanément selon le registre du dévoilement, ce qui fait d'elle à la fois le sens "donné" et le résultat du processus de mise en scène. Cette dualité n'est pas sans rapport avec la nouvelle ambiguïté qui atteint l'expression de «contenu interne": celui-ci est associé à la dialectique du jeu et trouve sa forme dans la mise en geste; mais il définit aussi l'image globale elle-même, considérée comme le thème produit, et se place par conséquent à l'issue du processus entier.

C'est que la notion d'image globale impose un troisième terme: le spectateur, qu'elle atteint intellectuellement et émotionnellement. Eisenstein ne le mentionne pas dans ce texte, sans doute parce que la mise en scène n'est que le premier stade destiné à subir l'emprise de la mise en cadre et du montage. L'image globale produite par la mise en scène sera elle-même incluse dans des processus dialectiques emboîtés qui la mèneront enfin, dans sa nouvelle forme, à produire un impact sur le spectateur. Car, si l'image est un contenu, un sens, elle est aussi à l'origine d'un effet. Alors réapparaît la dimension psychologique de l'intériorité - déplacée du côté de la réception ${ }^{19}$ - à laquelle ne se résume pourtant pas l'effet sur le spectateur puisqu'il faut compter encore sur l'aspect intellectuel. La notion d'image mène à une totalisation du phénomène et force à sortir la théorie de la mise en scène de la relation duelle et autonome de signification, pour se confronter ensuite aux autres stades de la réalisation. Même si le spectateur n'est pas explicitement nommé dans ce texte, l'image globale, propre au système eisensteinien, l'implique manifestement.

Nous avons suivi trois étapes de ce qu'Eisenstein appelle la mise en scène (voir figure 1). Le principe moteur premier était le motif profond propre à l'intériorité du personnage, à l'inconscient. Alors, l'« interne» était du côté de la cause, de l'origine; et nous avons vu sa complexification dans la mise en jeu à travers la dialectique des motifs. Dans le passage à la mise en geste est introduite une acception sémiologique de ce qui est caché ou interne, en accord avec la logique du dévoilement. Avec le troisième exemple, celui qui offre une vue d'ensemble de la mise en scène, 
Eisenstein dynamise le rapport sémiologique et le rend ambigu en introduisant une direction: le contenu, ce qui est interne, est à la fois une donnée de départ et un résultat. De plus, dans l'effet qu'il produit, il recrée de "l'émotion intérieure", cette fois chez le spectateur. Se juxtaposent alors deux manières de voir et de décrire le processus: celle qui, selon la logique du "dévoilement", présuppose un sens "déjà-là", caché; et celle qui implique une construction-élaboration de ce sens, et le projette comme troisième terme dans le spectateur, avec l'effet qui l'accompagne.

Le glissement dans l'emploi d' "interne» et les ambiguïtés qui en sont le prix pourraient sembler une faiblesse théorique. Ils sont pourtant la force du système de la mise en scène. Le motif psychologique de départ, l'intériorité des personnages, ce qui relevait de l'acte manqué, n'est plus comme tel l'essence de l'expressivité: il n'est plus ce que "voit" le spectateur ni ce qui l'atteint. Ce dernier est au contraire en face d'une image globale, qui a totalement transformé l'émotion du personnage en autre chose. Eisenstein refuse un certain mode d'expressivité, celui qui se fonde, pour toucher le spectateur, sur l'impact direct du motif intérieur psychologique, de l'émotion. L'intériorité ne peut ni ne doit "apparaître" comme telle sur l'acteur-personnage, pour être vue comme telle par le spectateur: ce serait se fier au modèle de Stanislavski, où l'être-là de l'émotion dans l'incarnation garantit l'effet de sa représentation. L'intériorité du personnage est bien essentielle pour Eisenstein, mais elle doit passer par une «machine à transformer" sa nature. Ce changement intervient à la faveur du processus dialectique qui englobe cette émotion première (du personnage) dans un système d'oppositions, mais aussi grâce au phénomène d'inclusion que la mise en scène développe en créant des niveaux dialectiques emboîtés où le sens d' «interne» change de nature au fur et à mesure qu'il s'applique au niveau le plus globalisant du système. Cette proposition théorique se distingue quelque peu de celle développée dans "Montage 1938 " par l'accent qu'elle met sur le processus de transformation. Si l'on en croit le texte de 1938, tout noyau du film, chacune de ses parties, doit renvoyer à l'image globale; il doit contenir ce thème unique. On pourrait croire qu'il n'y a pas de changement de nature entre la partie et le tout. De plus, le 
lien qui articule ces parties relève de la succession, de la juxtaposition ${ }^{20}$, sans qu'Eisenstein ne mentionne la dialectique impliquée dans la construction du tout. Or, justement, le glissement terminologique d'« intériorité" vers "contenu interne", puis " sens produit ", implique ce changement de fait. L'«interne" d'origine, est psychologique; il devient sémiologique, pour finir comme un processus dynamique à la fois sémiologique et psychologique, produit des stades précédents. Ce n'est qu’à travers ces stades - motif, action, mise en jeu, mise en geste, mise en scène -, que l'émotion gagne son pouvoir expressif, qu'elle ne peut assumer seule ni en tant que telle.

\section{Figure 2}

La mise en scène avec les différents éléments «internes».

Reconstitution théorique

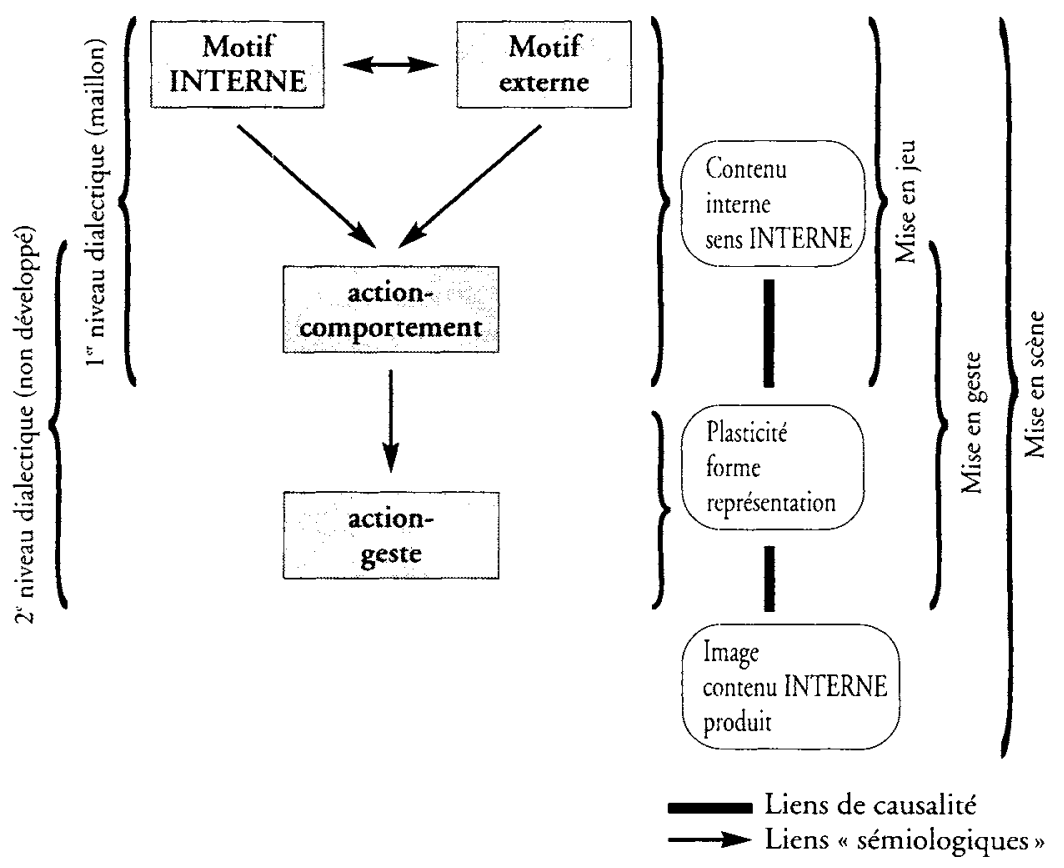

Université de Lausanne 


\section{NOTES}

1. François Albera parle à ce propos de "pratique théorique", qu'il distingue de "discours théorique", et montre que le principe dialectique qui les fonde l'une et l'autre est distinct. Voir Notes sur l'esthétique d'Eisenstein (Lyon: CIRS, 1973, p. 63).

2. Eisenstein parle d' "irruption en surface", ce qui est une forme d'apparition.

3. À propos de l'usage de la notion d'inconscient chez Stanislavski, Jean-Yves Pidoux précise: "Ces notations situent Stanislavski tout près d'une psychologie des profondeurs. De la notion d'intériorité telle qu'elle est conçue ici à celle d'inconscient, il n'y a qu'un pas. Stanislavski l'a franchi, mais non sans hésitation: sa connaissance de la psychologie était pratiquement excellente, mais théoriquement faible, de son propre aveu" dans Acteurs et personnages. L'Interprétation dans les esthétiques théâtrales du XX' siècle (Lausanne: L'Aire, 1986, p. 86).

4. Voir Constantin Stanislavski, La Formation de l'acteur (Paris: Petite Bibliorhèque Payot, 1982, p. 282-283, notamment).

5. François Albera remarque, dans son édition du texte, qu'Eisenstein transforme les termes de Freud: Fehlhandiung vient à la place de Fehlleistung, ce qui montre encore son intérêt pour l'action. Voir notes 2 et 3 (op. cit.), p. 277.

6. "Il n'y a là aucune "mystique". Mais un degré du processus même de l'hypnose. Car les actions hypnotiques permettent d" annihiler" les couches de la conscience, les centres ou zones cérébraux qui régularisent (stimulent, freinent, mobilisent en sens contraire, etc.) les manifestations automatiques directes. C'est-à-dire tout ce qui s'oppose à l'accomplissement automatique et inconditionnel des "ordres" de l'extérieur. "Le drilling [exercice] militaire vise surtout à faire disparaitre toute zone intermédiaire rationnelle entre le commandement et sa réalisation directe (réflexe)" (op. cit.), p.180.

7. Eisenstein aborde néanmoins la question dans "Montage 1938" (1958) où il décrit ce moment où le "sentiment s'empare de l'acteur " comme un effort volontaire de l'imagination. La méthode consiste "à obliger notre imagination à nous peindre une série de situations ou de tableaux concrets correspondant à notre thème. La somme de ces tableaux imaginaires suscite en réponse l'émotion, le sentiment, la souffrance, l'état d'âme cherchés" (p. 88). Limagination volontaire doit être strictement distinguée de la "mémoire affective" de Stanislavski. On ne revit pas une émotion passée, mais on crée le sentiment intérieur par la juxtaposition d'une série de tableaux imaginaires: en somme, l'acteur se fabrique son film intérieur.

8. À propos de la "négation de la négation ", voir "Il Ritorno del soldato dal fronte" (1989a, p. 89-90).

9. La voix du maitre Tortsov rend compte des positions de Stanislavski: "Ce qui intéresse le spectateur, ce n'est pas tant vos mouvements que ce qui se passe en vous. C'est votre vie intérieure, adaptée à votre rôle, qui doit animer la pièce. [...] C'était le processus normal et logique: l'expérience intérieure vient en premier, puis elle revêt une forme extérieure" (La Formation de l'acteur, op. cit., p. 168). Et encore: l'élève vient de réussir l'exercice du "revivre", il vient en somme de jouer avec sa propre émotion devant le mattre qui assumait la place du spectateur. Le texte continue ainsi: "Très bien, dit Tortsov. Vous vous êtes réellement libéré de toute tension. Maintenant je suis convaincu de chacun de vos gestes, et même si je ne sais pas exactement ce que vous avez dans l'esprit, je crois en vos pensées" (ibid., p. 286, nous soulignons). En somme, l'émotion "est là " et, de ce fait, garantit la croyance du spectateur en ce qui se passe devant ses yeux : c'est une relation d'immédiateté.

10. Laspect collectif est essentiel dans le travail de mise en scène, qui fait collaborer ensemble le metteur en scène et les acteurs. Voir "Le loup et l'agneau. Metteur en scène et acteur" (1958, p. 122-124). 
11. Les mêmes termes apparaissent un peu plus haut: "C'est ainsi que l'effort pour projeter un conflit interne en une forme tangible, un fait tangible, produit une situation de jeu - un maillon de jeu incarnant et dévoilant la cuisine interne des émotions de certains personnagges dans une certaine situation." (p. 184, c'est nous qui soulignons).

12. Remarquons à ce sujet le développement qu'Eisenstein donne à cet exemple. Après en avoir démontré le fonctionnement dialectique explicité ici, il le propose comme schéma de différentes variantes. Ainsi, il explique que la structure de base serait la même dans une intrigue romantique du genre "mélodrame du début du XIX siècle " (ibid.). Les traits seraient alors très accusés: la clef ne tourne plus fortuitement dans la serrure, car c'est explicitement que l'homme enferme la femme, pour la violenter; elle, exprime sa frayeur, «et c'est avec une venimeuse ironie que résonnent les paroles sur les propriétés des serrures dans la bouche grimaçante de l'intrigant aux lèvres fines s'avançant d'une démarche féline vers la malheureuse condamnée [...]". Le schéma est le même, en effet, pour ce qui concerne le jeu dialectique, mais il n'y a plus du tout d'intériorité cachée, de motif inconscient. Au contraire, tout est étalé au grand jour des intentions malfaitrices. Il est significatif - et quand même surprenant - que, toute problématique de l'intériorité cachée ayant disparu, rien ne change à la nature du schéma. Le privilège est radicalement du côté de l'enchaînement des actions, d'une logique de surface.

13. Il parle aussi de "comportement "inversé" " (p. 193) ou de "l'inversion" des actes" (p. 189), évidemment en référence à l'otkas meyerholdien.

14. Voir François Albera, "De L'Opinion publique à La Journaliste" dans: Vers une théorie de l'acteur. Acies du colloque de Lausanne (Lausanne: L'Âge d'Homme, 1994, p. 63-64).

15. L'ambiguïté est manifeste lorsque, dans la même page, il explicite à la fois le "contenu interne" et renvoie à l'intériorité des personnages: "Il est évident que la "coïncidence" passe non seulement par le contour le plus général et la disposition générale des personnages - tout ce qui est intérieur, du contenu affectif au degré de tension des muscles, s'oppose l'un à l'autre" (p. 194).

16. On retrouve cette ambiguïté dans la conclusion du deuxième exemple qui fait émerger l'intériorité du personnage, désignée cette fois par l'expression de "contenu interne ": "En conclusion de cetre analyse, nous dirons avoir cherché, dans la mesure de nos possibilités, à transformer le contenu interne des pensées et des sentiments du héros non seulement en actes qui les traduisent, mais en éléments formels à travers un mouvement [...], le mouvement même poursuivant et achevant la mise à nu de ce contenu interne" (p. 203)

17. À part sur ce point, Eisenstein affirme son désaccord avec les interprétations de cet auteur.

18. Et encore: "Il n'y a ici ni allégorie ni symbole. Mais une esquisse exacte des voies plastiquement déguisées en forme de trottoirs et trahissant par le contour de ce déguisement l'histoire toute en pulsations du cours des relations mutuelles des deux héros" (p. 205).

19. Nous retrouvons les positions d'Eisenstein dans les années vingt: l'émotion apparaît dans le public, dans le spectateur : voir "Le montage des attractions au cinéma" (1974) et "Le mouvement expressif" (1998, notamment p. 211).

20. Les occurrences sont très nombreuses: nous avons déjà cité "La juxtaposition de ces détails particuliers dans un certain mode de montage [...]", op. cit., p. 71. Voir encore notamment "Il fallait se tourner vers l'élément fondamental qui détermine également le contenu interne de chaque séquence et la juxtaposition de ces matériaux, c'est-à-dire vers le contenu du tout, de l'ensemble, de ce qui rassemble" (p. 70). 


\section{OUVRAGES CITÉS}

N.B.: les ouvrages cités d'Eisenstein se retrouvent, en fin de numéro, dans une liste particulière.

252 CiNéMAS, vol. $11, n^{\text {os }} 2-3$ 